REVISTA INTERNACIONAL DE CIENCIAS DEL DEPORTE International Journal of Sport Science

Rev. int. cienc. deporte

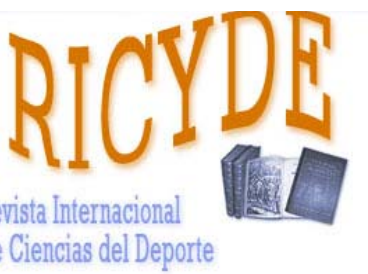

International Journal of Sport Science VOLUMEN IX - AÑO IX

Páginas:181-199 ISSN:1885-3137 No 32 - Abril - 2013

\title{
Beneficios del Tai Chi Chuan en la osteoartritis, el equilibrio y la calidad de vida Tai Chi Chuan benefits on osteoarthritis, balance and quality of life
}

\author{
Pedro Jesús Jiménez-Martín, Agustín Meléndez-Ortega \\ Universidad Politécnica de Madrid, España \\ Ulrike Albers \\ Korea Advanced Institute of Science \& Technology, Corea del Sur \\ Alfonso López-Díaz \\ Universidad Politécnica de Madrid, España
}

\section{Resumen}

Objetivo: Este trabajo hace una revisión de los estudios científicos realizados a nivel internacional con el fin de actualizar el conocimiento sobre cuáles son los beneficios que aporta la práctica del Tai Chi Chuan (TCC) sobre la osteoartritis de rodilla (OR), el equilibrio, el miedo a las caídas y la calidad de vida. Método: Se realizó una búsqueda bibliográfica sobre ocho bases de datos internacionales (Medline-Pubmed, Scirus, Pascal, ScienceDirect, SportDiscus, Science Citation Index, BIOSIS y PsycINFO) hasta abril del año 2012. Resultados: El número total de estudios analizados fue de 74, de los cuales 13 corresponden a revisiones sistemáticas y 61 a estudios específicos (42 ensayos aleatorios controlados (EAC) y 19 ensayos no controlados (ENC). En particular se analizaron 11 estudios con relación a la osteoartritis de rodilla (7 EAC y 4 ENC), 32 sobre el equilibrio-miedo a las caídas (26 EAC y 6 ENC), y 30 sobre calidad de vida (18 EAC y 12 ENC) Conclusión: Los efectos de TCC sobre la OA son contradictorios, aunque se observa una tendencia positiva respecto al control de dolor y la mejora en la funcionalidad física. Respecto a los efectos sobre el equilibrio-miedo a la caída se ha registrado una mejora significativa en el equilibrio estático y dinámico, y en la confianza respecto al miedo a la caída. Con relación a la calidad de vida la práctica del TCC aporta mejoras en las dimensiones de bienestar emocional, percepción de sí mismo, bienestar corporal. Estos resultados obtenidos deben ser tomados con cierta precaución dado que gran número de los estudios examinados muestran carencias metodológicas en cuanto al diseño de investigación (muestras reducidas, carencias en los criterios de inclusión y de exclusión de los participantes, deficiencias en los análisis estadísticos, carencias en la estandarización de la medición de resultados, etc.) y la información específica respecto a las condiciones del programa de TCC utilizado (el estilo practicado, la dosificación, la experiencia de los instructores, la altura en las posturas, el tiempo de ejecución de cada forma, etc.).

Palabras clave: Tai Chi Chuan; osteoartritis; equilibrio; miedo a la caída; calidad de vida.

\section{Abstract}

Aim: This paper reviews international scientific studies aimed at determining the benefits of practicing Tai Chi Chuan (TCC) on knee osteoarthritis (OR), balance, fear of falling, and quality of life. Method: A bibliographic search of eight international databases was undertaken (Medline-Pubmed, Scirus, Pascal, ScienceDirect, SportDiscus, Science Citation Index, BIOSIS y PsycINFO) until April 2012. Results: A total of 74 studies were analyzed, 13 of them corresponding to systematic reviews and 61 to specific studies (42 randomized controlled studies (EAC) and 19 non-controlled studies (ENC)). In particular, 11 studies were analyzed regarding knee osteoarthritis (7 EAC and 4 ENC), 32 regarding balance-fear of falling ( 26 EAC and 6 ENC), and 30 regarding quality of life (18 EAC and 12 ENC). Conclusion: The effects of TCC on knee osteoarthritis are contradictory although a positive tendency was observed regarding control of pain and the improvement of physical functionality. Regarding the effects on balance-fear of falling, a significant improvement in static and dynamic balance was observed as well as confidence in terms of fear of falling. Regarding quality of life, TCC practice improves emotional well-being, self-perception and physical well-being. The obtained results should be treated with some caution since many of the studies examined show methodological weaknesses in the design (small samples, lack of inclusion and exclusion criteria for the participants, deficiencies in the statistical analyses, lack of standardization in the measurement of results, etc.), and the specific information about the conditions of the TCC program applied (style practised, dose, experience of the instructors, posture height, time spent carrying out each form).

Key words: Tai Chi Chuan; osteoarthritis; balance; fear of falling; quality of life.

Correspondencia/correspondence: Pedro Jesús Jiménez-Martín

Facultad de Ciencias de la Actividad Física y del Deporte. Universidad Politécnica de Madrid, España

Calle Martín Fierro, no 7, 28040. Madrid España

E-mail: pedrojesus.jimenez@upm.es 
Jiménez-Martín, P.J.; Meléndez-Ortega, A.; Albers, U.; López-Díaz, A. (2013). Beneficios del Tai Chi Chuan en la osteoartritis, el equilibrio y la calidad de vida. RICYDE. Rev. int. cienc. deporte. 32(9), 181-199. http://dx.doi.org/10.5232/ricyde2013.03206

\section{Introducción}

$\mathrm{L}$ a osteoartritis (OA) es una patología crónica que afecta a las articulaciones (más a menudo a rodillas, caderas y dedos) y que causa entre otros aspectos dolor, limitación funcional y rigidez (Lee, Park, Chae, Kim, Kim, Kim, Kim, Yin, y Lee, 2009). Actualmente no existe una cura para este problema de modo que la estrategia terapéutica que se ha adoptado es de carácter sintomático (Vignon, Valat, Rossignol, Avouac, Rozenberg, Thoumie, Avouac, Nordin, y Hilliquin, 2006). Dentro del ámbito de la actividad física y del deporte, el Tai Chi Chuan (TCC) como una actividad de baja-mediana intensidad se está considerando como una terapia de ejercicio alternativa frente a esta patología.

Las caídas, en personas mayores de 65 años suponen, además de un gran coste para la sanidad pública, un gran problema por la pérdida de la calidad de vida que afecta a este perfil poblacional. Uno de los factores básicos asociados a las caídas es el deterioro en el control del equilibrio que se produce en los ancianos, fruto de la degeneración que padecen en los sistemas propioceptivos y motores (Aparicio, Carbonell-Baeza y Delgado-Fernández, 2010). Dentro de los programas de actividad física y deporte que se están utilizando para potenciar el equilibrio y evitar el riesgo de caída en ancianos, el TCC también se ha convertido en un referente de investigación para numerosos autores a nivel internacional.

A nivel psicológico, el TCC también se han convertido en centro de atención en dos aspectos: su influencia en la mejora de la función cognitiva y sobre la calidad de vida. Si bien el primero todavía no ha recibido suficiente número de estudios (Chang, Nien, Tsai, y Etnier, 2010), el segundo, cuenta con numerosos estudios específicos, como se podrá comprobar en este trabajo.

El TCC es una práctica física de salud en forma de coreografía procedente de China cuyos orígenes se perfilan en la tradición taoísta y que engloba los mismos principios de la medicina tradicional china. Desde el siglo XVII, en que adopta su forma moderna, se han ido creado diferentes escuelas o estilos siendo los más importantes: Chen, Yang, Wu, Hao y Sun. Aunque estos estilos comparten la misma visión filosófica, cada uno de ellos muestra una personalidad propia: los estilos Yang, $\mathrm{Wu}$, Hao y Sun se caracterizan por realizar movimientos circulares lentos y continuos e incorporar elementos de equilibrio, respiración natural, alineamiento postural, coordinación, relajación y concentración. El estilo Chen, más cercano a un concepto marcial, se desarrolla en posiciones más bajas, con cambios de ritmo que incluyen movimientos explosivos, saltos y rotación articular (Chang y col. 2010; Chen, Chen, y Huang, 2006; Hall y col., 2009a; Low, Ang, Goh, y Chew, 2009; Rogers, Larkey, y Keller 2009; Wang, Collet y Lau, 2004; Wang, Raveendhara, Ramel, Kupelnick, Scott, y Schmid, 2010; Yeh, Wang, Wayne, y Phillipis, 2009).

El objetivo de este estudio es hacer una revisión sobre cuáles son los ámbitos de estudio y los efectos de la práctica del TCC sobre sus practicantes en tres aspectos: osteoartritis, equilibriomiedo a la caída y la calidad de vida, tomando como referencia los estudios científicos de revisión sistemática y específicos realizados. La elección de estos parámetros responde fundamentalmente a que estos factores representan una de las principales problemáticas que afectan a la salud de los ancianos. 
Jiménez-Martín, P.J.; Meléndez-Ortega, A.; Albers, U.; López-Díaz, A. (2013). Beneficios del Tai Chi Chuan en la osteoartritis, el equilibrio y la calidad de vida. RICYDE. Rev. int. cienc. deporte. 32(9), 181-199. http://dx.doi.org/10.5232/ricyde2013.03206

\section{Material y método}

Para localizar los estudios analizados en este trabajo se utilizaron 8 bases de datos (MedlinePubmed, Scirus, Pascal, ScienceDirect, SportDiscuss, Science Citation Index, BIOSIS y PsycINFO) y se eligieron como palabras clave diferentes combinaciones con los términos: Tai Chi, T’ai Chi, Tai Ji, Tai Chi Chuan, Tai Ji Quan, OA, osteoarthritis, KOA, knee osteoarthritis, balance, falls, fear of falls, psychology, cognition, stress, anxiety, depression, mood, sleep disorder, well-being, self-efficacy, mental health, self-esteem y quality of life. La revisión se realizó hasta abril del año 2012. Como criterio de inclusión para seleccionar los estudios se decidió que estuviesen escritos en inglés o en castellano, y como criterio de exclusión se desechó a todos aquellos que: no tuviesen como objetivo la medición de los efectos del TCC sobre las patologías seleccionadas, fuesen actas de congreso, resúmenes, pósters, o artículos repetidos o redundantes o artículos que mezclasen el TCC con prácticas de Qigong.

La información extraída de los diferentes estudios se estructuró en tablas en los siguientes apartados: autor(es), año de publicación, diseño del estudio, país en el que se efectuó la intervención, estilo de TCC y el número de movimientos de la forma utilizada, duración y frecuencia de la intervención, tamaño muestral, diferenciando género y media de edad, y resultados obtenidos. En el análisis sobre los efectos psicológicos del TCC se optó, ante la falta de claridad en la clasificación y definición encontrada de los aspectos que engloba la calidad de vida en los estudios, por utilizar el modelo elaborado por Spirduso, Francis, y MacRae (2005) que define y clasifica las variables que conforman la calidad de vida.

\section{Resultados}

Del total de 16.753 artículos encontrados en las bases de datos analizadas, el número total de estudios analizados que cumplieron los criterios de inclusión para este estudio fue de 73 , de los cuales 13 corresponden a revisiones sistemáticas ( 3 de ellas también realizaron un metaanálisis de los datos) y 61 a estudios específicos (42 ensayos aleatorios controlados (EAC) y 19 ensayos no controlados (ENC)). Dentro de los estudios específicos, 11 analizaron los efectos del TCC sobre la osteoartritis de rodilla (7 EAC y 4 ENC), 32 sobre el equilibriomiedo a las caídas (26 EAC y 6 ENC) y 30 la calidad de vida (18 EAC y 12 ENC). Las investigaciones se llevaron a cabo entre los años 1989-2012 y los países donde se realizaron corresponden a Estados Unidos (34 estudios), China (14), Corea (3), Australia (4), Francia (2), España (1), Japón (1), Canadá (1) e Inglaterra (1).

Los estilos de TCC utilizados en las intervenciones fueron el Yang (37 estudios) en versiones simplificadas de 5, 8, 9, 10, 12, 15, 18 y 24 movimientos, y sólo 2 estudios utilizaron el sistema tradicional de 108 movimientos; el Sun de 12 movimientos (5), el Wu (3) y el Chen (1). La duración de las intervenciones oscila entre 3 semanas y 2 años, pero anulando estos extremos, la media ponderada es de 16.5 semanas y la moda 12 semanas. La media en la frecuencia de clases corresponde a 2.4 veces por semana y la duración 55 minutos por sesión.

En las muestras, los estudios en el que el número de sujetos que practican TCC en la intervención está por debajo de 20 es de 26, y por encima 35. El rango oscila entre 2 y 83 personas, pero anulando estas puntaciones la media ponderada es de 28 participantes. Respecto al género, el número de estudios en el que hay mayor número de mujeres que de varones es de 26, y al contrario de 19. Con relación a la edad, los estudios con una media de edad inferior a 55 años son 13 y por encima 46. En la Tabla 1 se muestra un resumen de estos datos. 
Jiménez-Martín, P.J.; Meléndez-Ortega, A.; Albers, U.; López-Díaz, A. (2013). Beneficios del Tai Chi Chuan en la osteoartritis, el equilibrio y la calidad de vida. RICYDE. Rev. int. cienc. deporte. 32(9), 181-199. http://dx.doi.org/10.5232/ricyde2013.03206

Tabla 1.- Resumen de los estudios que analizados

\begin{tabular}{|c|c|c|c|c|c|c|c|c|}
\hline Estudios & Diseño & País & $\mathbf{n}$ & Género & Edad & $\begin{array}{c}\text { Estilo TCC } \\
\text { y n }^{\circ} \text { mov }\end{array}$ & $\begin{array}{c}\text { Duración } \\
\text { (Semanas) }\end{array}$ & Frecuencia \\
\hline Audette y col. (2006) & EAC & EE.UU & 11 & i? i?m & $71.5( \pm 4.6)$ & Yang 10 & 12 & $3 \mathrm{v} / \mathrm{s}-1 \mathrm{~h} / \mathrm{s}$ \\
\hline Barnett y col.. (2003) & EAC & Australia & 83 & $25 \mathrm{v} 58 \mathrm{~m}$ & $74.4( \pm 4.9)$ & $i ?$ & 52 & $i ?-1 \mathrm{~h} / \mathrm{s}$ \\
\hline Baron y Faubert (2005) & ENC & Canadá & 3 & $2 \mathrm{v} 1 \mathrm{~m}$ & 13.3 & Yangi? & 10 & $2 \mathrm{v} / \mathrm{s}-1 \mathrm{~h} / \mathrm{s}$ \\
\hline Blake y Batsonv(2009) & EAC & Inglaterra & 10 & $9 \mathrm{v} 1 \mathrm{~m}$ & $44.5( \pm 10.52)$ & Yang 18 & 8 & $1 \mathrm{v} / \mathrm{s}-1 \mathrm{~h} / \mathrm{s}$ \\
\hline Brismée y col. (2007) & EAC & EE.UU & 18 & $3 \mathrm{v} 19 \mathrm{~m}$ & $70.8( \pm 9.8)$ & Yang 24 & 12 & $3 \mathrm{v} / \mathrm{s}-40 \mathrm{~m} / \mathrm{s}$ \\
\hline Brown y col. (1995) & EAC & EE.UU & 18 & $11 \mathrm{v} 7 \mathrm{~m}$ & 50.75 & ¿? & 16 & $1 \mathrm{v} / \mathrm{s}-45 \mathrm{~m} / \mathrm{s}$ \\
\hline Chen y col. (2008) & ENC & China & 13 & $7 \mathrm{v} 6 \mathrm{~m}$ & 68.5 & Sun 12 & 104 & $3 \mathrm{v} / \mathrm{s}-50 \mathrm{~m} / \mathrm{s}$ \\
\hline Deschamps y col. (2009) & EAC & Francia & 26 & $18 \mathrm{v} 8 \mathrm{~m}$ & $80.8( \pm 8.7)$ & Yang ${ }_{i} ?$ & 24 & $4 \mathrm{v} / \mathrm{s}-30 \mathrm{~m} / \mathrm{s}$ \\
\hline Ding-Hai y Hui-Xin (2012) & ENC & China & 38 & $38 \mathrm{v} 0 \mathrm{~m}$ & $59.7( \pm 5.6)$ & Yang 24 y 42 & 24 & $3 \mathrm{v} / \mathrm{s}-1 \mathrm{~h} / \mathrm{s}$ \\
\hline Field y col. (2010) & ENC & EE.UU & 38 & $2 \mathrm{v} 36 \mathrm{~m}$ & $41( \pm 38)$ & ¿? & ¿? & $i ? \mathrm{v} / \mathrm{s}-i ? \mathrm{~h} / \mathrm{s}$ \\
\hline Fong y $\mathrm{Ng}(2006)$ & ENC & China & 32 & $19 \mathrm{v} 13 \mathrm{~m}$ & $53.8( \pm 12.1)$ & ¿? & ¿? & $i ? \mathrm{v} / \mathrm{s}-\mathrm{h} / \mathrm{s}$ \\
\hline Fransen y col. (2007) & EAC & EE.UU & 56 & $18 \mathrm{v} 38 \mathrm{~m}$ & $70.8( \pm 6.3)$ & Sun 12 & 12 & $3 \mathrm{v} / \mathrm{s}-1 \mathrm{~h} / \mathrm{s}$ \\
\hline Galantino y col. (2005) & EAC & EE.UU & 13 & $i ? \mathrm{v} i ? \mathrm{~m}$ & ¿? & ¿? & 8 & $2 \mathrm{v} / \mathrm{s}-50 \mathrm{~m} / \mathrm{s}$ \\
\hline Gatts y Woollacott (2007) & EAC & EE.UU & 11 & $1 \mathrm{v} 10 \mathrm{~m}$ & 77.5 & Yang 12 & 3 & $5 \mathrm{v} / \mathrm{s}-1.5 \mathrm{~h} / \mathrm{s}$ \\
\hline Gyllensten y col. (2010) & EAC & China & 24 & $i ? \mathrm{v} i ? \mathrm{~m}$ & $68.5( \pm 6.6)$ & Yang 24 & ¿? & $i ? \mathrm{v} / \mathrm{s}-i ? \mathrm{~h} / \mathrm{s}$ \\
\hline Hackney y Earhart (2008) & EAC & EE.UU & 13 & $11 \mathrm{v} 2 \mathrm{~m}$ & $64.9( \pm 8.3)$ & Yang 12 & 13 & $2 \mathrm{v} / \mathrm{s}-1 \mathrm{~h} / \mathrm{s}$ \\
\hline Hain y col. (1999). & ENC & EE.UU & 22 & $i ? \mathrm{v} i ? \mathrm{~m}$ & $20-76$ & Yang-Wu 8 & 8 & $1 \mathrm{v} / \mathrm{s}-1 \mathrm{~h} / \mathrm{s}$ \\
\hline Hall y col. (2009b) & EAC & EE.UU & 8 & $i ? \mathrm{v}_{i} ? \mathrm{~m}$ & $72.2( \pm 7.7)$ & Yang 24 & 12 & $2 \mathrm{v} / \mathrm{s}-1.5 \mathrm{~h} / \mathrm{s}$ \\
\hline Hartman y col. (2000) & EAC & EE.UU & 18 & $3 \mathrm{v} 15 \mathrm{~m}$ & $68.6( \pm 7.9)$ & Yang 9 & 12 & $2 \mathrm{v} / \mathrm{s}-1 \mathrm{~h} / \mathrm{s}$ \\
\hline Hernádez-Reif y col. (2001) & ENC & EE.UU & 13 & $11 \mathrm{v} 2 \mathrm{~m}$ & 14.5 & ¿? & 5 & $2 \mathrm{v} / \mathrm{s}-30 \mathrm{~m} / \mathrm{s}$ \\
\hline Hoffmann y col. (2009) & ENC & EE.UU & 19 & $5 \mathrm{v} 14 \mathrm{~m}$ & 52 & Yang 24 & 10 & $2 \mathrm{v} / \mathrm{s}-1 \mathrm{~h} / \mathrm{s}$ \\
\hline Irwin y col. (2008) & EAC & EE.UU & 59 & $18 \mathrm{v} 41 \mathrm{~m}$ & $69.8( \pm 6.2)$ & ¿?20 & 25 & $3 \mathrm{v} / \mathrm{s}-40 \mathrm{~m} / \mathrm{s}$ \\
\hline $\operatorname{Jin}(1989)$ & EAC & Australia & 33 & $20 \mathrm{v} 13 \mathrm{~m}$ & $37.7( \pm 14.3)$ & Yang108/Wuic? & ¿? & $i ? \mathrm{v} / \mathrm{s}-i ? \mathrm{~h} / \mathrm{s}$ \\
\hline $\operatorname{Jin}(1992)$ & EAC & Australia & 48 & $48 \mathrm{v} 0 \mathrm{~m}$ & $34.6( \pm 8.5)$ & Yang108/Wu $\mathrm{Wu}_{\mathrm{i}}$ ? & ¿? & $i ? \mathrm{v} / \mathrm{s}-i ? \mathrm{~h} / \mathrm{s}$ \\
\hline Lee y col. (2009) & EAC & Corea & 28 & $2 \mathrm{v} 26 \mathrm{~m}$ & $70.2( \pm 4.8)$ & TCQ 18 & 8 & $2 \mathrm{v} / \mathrm{s}-1 \mathrm{~h} / \mathrm{s}$ \\
\hline Lelard y col . (2010) & EAC & Francia & 14 & $i ? \mathrm{v} i ? \mathrm{~m}$ & $76.8( \pm 5.1)$ & $i ? 12$ & 12 & ¿? $? \mathrm{v} / \mathrm{s}-30 \mathrm{~m} / \mathrm{s}$ \\
\hline Li y col. (2001) & EAC & EE.UU & 49 & $i ? \mathrm{v} i ? \mathrm{~m}$ & $72.8( \pm 4.7)$ & Yang 24 & 24 & $2 \mathrm{v} / \mathrm{s}-1 \mathrm{~h} / \mathrm{s}$ \\
\hline Li y col. (2004) & EAC & EE.UU & 62 & $10 \mathrm{v} 52 \mathrm{~m}$ & $75.3( \pm 7.8)$ & Yang 8 & 24 & $3 \mathrm{v} / \mathrm{s}-1 \mathrm{~h} / \mathrm{s}$ \\
\hline Li y col. (2012) & EAC & EE.UU & 65 & $45 \mathrm{v} 20 \mathrm{~m}$ & $68( \pm 9)$ & ¿? 6 & 24 & $2 \mathrm{v} / \mathrm{s}-1 \mathrm{~h} / \mathrm{s}$ \\
\hline Liu y col. (2008) & ENC & Australia & 11 & $3 \mathrm{v} 8 \mathrm{~m}$ & 53.5 & KaiMai $\_?$ & 12 & $3 \mathrm{v} / \mathrm{s}-1 \mathrm{~h} / \mathrm{s}$ \\
\hline Mark y Ling (2003) & EAC & China & 19 & $i ? \mathrm{v} i ? \mathrm{~m}$ & $61.8( \pm 4.7)$ & Yang 24 & 52 & $3 \mathrm{v} / \mathrm{s}-30 / 45 \mathrm{~m} / \mathrm{s}$ \\
\hline McGibbon y col. (2005) & EAC & EE.UU & 19 & $i ? \mathrm{v} i ? \mathrm{~m}$ & $61.7( \pm 11.3)$ & Yang 5 & 10 & $1 \mathrm{v} / \mathrm{s}-70 \mathrm{~m} / \mathrm{s}$ \\
\hline Ni y col. (2010) & EAC & China & 14 & $0 \mathrm{v} 14 \mathrm{~m}$ & $62.8( \pm 2.79)$ & Yang 24 & 24 & $2 \mathrm{v} / \mathrm{s}-40 \mathrm{~m} / \mathrm{s}$ \\
\hline Qin y col. (2005) & EAC & China & 48 & $0 \mathrm{v} 48 \mathrm{~m}$ & $55.8( \pm 3.5)$ & ¿? & ¿? & $i ? \mathrm{v} / \mathrm{s}-i ? \mathrm{~h} / \mathrm{s}$ \\
\hline Ramachandran y col. (2007) & EAC & EE.UU & 15 & $8 \mathrm{v} 7 \mathrm{~m}$ & $45.7( \pm 10.6)$ & Chen $_{i}$ ? & ¿? & $i ? \mathrm{v} / \mathrm{s}-i ? \mathrm{~h} / \mathrm{s}$ \\
\hline Reid-Arndt y col. (2011) & ENC & EE.UU & 33 & $i ? \mathrm{v} i ? \mathrm{~m}$ & $62.3( \pm 10.8)$ & Yang 15 & 10 & $2 \mathrm{v} / \mathrm{s}-1 \mathrm{~h} / \mathrm{s}$ \\
\hline Robins y col. (2006) & EAC & EE.UU & 59 & $35 \mathrm{v} 24 \mathrm{~m}$ & $42.3( \pm 8.3)$ & Yang 8 & 10 & ¿? \\
\hline Shen y col. (2008) & ENC & EE.UU & 40 & $4 \mathrm{v} 36 \mathrm{~m}$ & $64.4( \pm 8.3)$ & Yang 24 & 6 & $2 \mathrm{v} / \mathrm{s}-1 \mathrm{~h} / \mathrm{s}$ \\
\hline Song y col. (2003) & EAC & Corea & 22 & $0 \mathrm{v} 22 \mathrm{~m}$ & $64.8( \pm 6.0)$ & Sun 12 & 12 & $3 \mathrm{v} / \mathrm{s}-50 \mathrm{~m} / \mathrm{s}$ \\
\hline Song y col. (2007) & ENC & Corea & 13 & $7 \mathrm{v} 6 \mathrm{~m}$ & 68.5 & Sun 12 & 104 & $3 \mathrm{v} / \mathrm{s}-50 \mathrm{~m} / \mathrm{s}$ \\
\hline Soto y col. (2008) & ENC & España & 66 & $12 \mathrm{v} 54 \mathrm{~m}$ & $79.7( \pm 5.7)$ & Yang 10 & 12 & $2 \mathrm{v} / \mathrm{s}-1 \mathrm{~h} / \mathrm{s}$ \\
\hline Taggart (2002) & ENC & EE,UU & 45 & $0 \mathrm{v} 45 \mathrm{~m}$ & +65 & ¿? & 12 & $2 \mathrm{v} / \mathrm{s}-30 \mathrm{~m} / \mathrm{s}$ \\
\hline Taylor y Froelicher (2004) & ENC & EE.UU & 18 & $5 \mathrm{v} 13 \mathrm{~m}$ & $60( \pm 18.4)$ & ¿? & ¿? & ¿?v/s-i?h/s \\
\hline Taylor y Coull (2012) & EAC & EE.UU & 16 & $10 \mathrm{v} 6 \mathrm{~m}$ & $72.8( \pm 10.1)$ & Yang 24 & 12 & $3 \mathrm{v} / \mathrm{s}-1 \mathrm{~h} / \mathrm{s}$ \\
\hline Taylor y col. (2006) & ENC & EE.UU & 39 & $12 \mathrm{v} 27 \mathrm{~m}$ & $66( \pm 8.3)$ & Yang 24 & 12 & $3 \mathrm{v} / \mathrm{s}-1 \mathrm{~h} / \mathrm{s}$ \\
\hline Taylor y col. (2010) & EAC & EE.UU & 37 & $13 \mathrm{v} 24 \mathrm{~m}$ & $70.6( \pm 5.9)$ & Yang 24 & 24 & ¿? $?-45 \mathrm{~m} / \mathrm{s}$ \\
\hline Thorton y col. (2004) & EAC & China & 17 & 0v $17 \mathrm{~m}$ & $47.2( \pm 4.07)$ & Yang 108 & 12 & $3 \mathrm{v} / \mathrm{s}-1 \mathrm{~h} / \mathrm{s}$ \\
\hline Toda y col. (2011) & ENC & Japón & 22 & $0 \mathrm{v} 22 \mathrm{~m}$ & 68.9 & Yang & ¿? & $i ? \mathrm{v} / \mathrm{s}-i ? \mathrm{~h} / \mathrm{s}$ \\
\hline Tsai y col. (2009) & ENC & EE.UU & 6 & $0 \mathrm{v} 6 \mathrm{~m}$ & $83( \pm 6)$ & Sun 12 & 15 & $2 \mathrm{v} / \mathrm{s}-40 \mathrm{~m} / \mathrm{s}$ \\
\hline Tsang y col. (2004) & EAC & China & 20 & $10 \mathrm{v} 10 \mathrm{~m}$ & $70.7( \pm 5.1)$ & $\mathrm{NG}_{i}$ ? & 104 & $2 \mathrm{v} / \mathrm{s}-1 \mathrm{~h} / \mathrm{s}$ \\
\hline Tsang y Hui-Chan (2006) & EAC & China & 24 & $12 \mathrm{v} 12 \mathrm{~m}$ & $69.3( \pm 5.0)$ & $i ?$ & 4 & $i ? \mathrm{v} / \mathrm{s}-i ? \mathrm{~h} / \mathrm{s}$ \\
\hline Venglar (2005) & ENC & EE.UU & 2 & $1 \mathrm{v} 1 \mathrm{~m}$ & 58 & Yangi? & 8 & $1 \mathrm{v} / \mathrm{s}-1 \mathrm{~h} / \mathrm{s}$ \\
\hline Wang y col. (2009) & EAC & EE.UU & 20 & $4 \mathrm{v} 16 \mathrm{~m}$ & $63( \pm 8.1)$ & Yang 10 & 12 & $2 \mathrm{v} / \mathrm{s}-1 \mathrm{~h} / \mathrm{s}$ \\
\hline Wolf y col. (1996) & EAC & EE.UU & 64 & $12 \mathrm{v} 52 \mathrm{~m}$ & $76.9( \pm 4.8)$ & Yang 10 & 15 & $i ?-45 \mathrm{~m} / \mathrm{s}$ \\
\hline Wong y col. (2001) & EAC & China & 42 & $7 \mathrm{v} 17 \mathrm{~m}$ & $66.4( \pm 9)$ & ¿? & ¿? & $i ? \mathrm{v} / \mathrm{s}-i ? \mathrm{~h} / \mathrm{s}$ \\
\hline Woo y col. (2007) & EAC & China & 30 & $i ? \mathrm{v} i ? \mathrm{~m}$ & $68.2( \pm 2.4)$ & Yang 24 & ¿? & $1 \mathrm{~A}(3 \mathrm{v} / \mathrm{s}-i ?)$ \\
\hline Wu y col. (2010) & EAC & EE.UU & 64 & ¿? $\mathrm{v} i ? \mathrm{~m}$ & +65 & Yang 24 & 15 & $3 \mathrm{v} / \mathrm{s}-1 \mathrm{~h} / \mathrm{s}$ \\
\hline Xu, Hong y Chan (2004) & EAC & China & 21 & $12 \mathrm{v} 9 \mathrm{~m}$ & $66.1( \pm 5.2)$ & ¿? & ¿? & $i ? \mathrm{v} / \mathrm{s}-i ? \mathrm{~h} / \mathrm{s}$ \\
\hline Yan (1998) & EAC & EE.UU & 28 & ¿? $i ? \mathrm{~m}$ & $78.8( \pm 2.1)$ & Yang 24 & 8 & $3 \mathrm{v} / \mathrm{s}-45 \mathrm{~m} / \mathrm{s}$ \\
\hline Yeh y col. (2008). & EAC & EE.UU & 8 & $4 \mathrm{v} 4 \mathrm{~m}$ & $64.2( \pm 16.2)$ & Yang 5 & 12 & $2 \mathrm{v} / \mathrm{s}-1 \mathrm{~h} / \mathrm{s}$ \\
\hline Zhang y col. (2006) & EAC & China & 24 & $12 \mathrm{v} 12 \mathrm{~m}$ & $70.2( \pm 3.6)$ & Yang2 4 & 8 & $7 \mathrm{v} / \mathrm{s}-1 \mathrm{~h} / \mathrm{s}$ \\
\hline
\end{tabular}

Nota: $\mathrm{EAC}=$ Ensayos aleatorio controlado; $\mathrm{ENC}=$ Ensayo no controlado; $\mathrm{n}=$ muestra; $\mathrm{v}=$ varones, $\mathrm{m}=$ mujeres; $\mathrm{v} / \mathrm{s}=$ veces por semana; $\mathrm{h} / \mathrm{s}=$ horas por sesión; $\mathrm{m} / \mathrm{s}=$ minutos por sesión;; $i ?=$ sin especificar. 
Jiménez-Martín, P.J.; Meléndez-Ortega, A.; Albers, U.; López-Díaz, A. (2013). Beneficios del Tai Chi Chuan en la osteoartritis, el equilibrio y la calidad de vida. RICYDE. Rev. int. cienc. deporte. 32(9), 181-199. http://dx.doi.org/10.5232/ricyde2013.03206

\section{Osteoartritis}

El número de trabajos analizados con relación a la osteoartritis de rodilla fue de 11 (7 EAC y 4 ENC). Según estos estudios, el TCC produjo mejoras significativas en sus practicantes en la percepción de la funcionalidad física, la rigidez y el dolor. En la tabla 2 se muestra un resumen de los resultados.

Tabla 2.- Resultados de los estudios con relación a la osteoartritis de rodilla

\begin{tabular}{|c|c|c|c|c|c|c|}
\hline Estudios & Dolor & Rigidez & PDPF & $\begin{array}{l}\text { Rango } \\
\text { Mov. }\end{array}$ & $\begin{array}{c}\text { Subir } \\
\text { escaleras }\end{array}$ & $\begin{array}{c}\text { Tiempo } \\
\text { Caminando }\end{array}$ \\
\hline Brismée y col. (2007) & $(\mathrm{p}<0.05)$ & $(p=0.206)$ & $(\mathrm{p}=0.0075)$ & - & - & - \\
\hline Chen y col. (2008) & NS & MNS & $(\mathrm{p}<0.05)$ & - & - & - \\
\hline Fransen y col. (2007) & $\operatorname{MS}(i ?)$ & $\operatorname{MS}(i ?)$ & NS & - & - & - \\
\hline Hartman y col. (2000) & NS & - & $(\mathrm{p}<0.05)$ & - & MNS & MNS \\
\hline Lee y col. (2009) & MNS & MNS & MNS & - & - & $(\mathrm{p}=0.005)$ \\
\hline Ni y col. (2010) & $(\mathrm{p}=0.001)$ & $(\mathrm{p}=0.043)$ & $(\mathrm{p}=0.001)$ & - & $(p=0.003)$ & $(\mathrm{p}=0.001)$ \\
\hline Shen y col. (2008) & $(p=0.002)$ & $(\mathrm{p}=0.001)$ & $(\mathrm{p}<0.001)$ & - & - & $(\mathrm{p}<0.025)$ \\
\hline Song y col. (2003) & $(\mathrm{p}=0.034)$ & $(\mathrm{p}=0.039)$ & $(\mathrm{p}<0.01)$ & NM & - & - \\
\hline Song y col. (2007) & - & - & $(\mathrm{p}<0.05)$ & - & - & - \\
\hline Tsai y col. (2009) & MNS & - & - & - & - & - \\
\hline Wang y col. (2009) & $(\mathrm{p}=0.0005)$ & MNS & $(\mathrm{p}=0.001)$ & - & $(p=0.00005)$ & MNS \\
\hline Total que analizan & $10(100 \%)$ & $8(100 \%)$ & $10(100 \%)$ & $1(100 \%)$ & $3(100 \%)$ & $5(100 \%)$ \\
\hline Total MS & $5(50.0 \%)$ & $5(62.5 \%)$ & $8(80.0 \%)$ & $0(0.0 \%)$ & $2(66.6 \%)$ & $3(50.0 \%)$ \\
\hline
\end{tabular}

Nota: $M S=$ Mejora significativa, $M N S=$ Mejora no significativa; NS= No mejora; $₫ ?=$ Sin especificar.

\section{Equilibrio y miedo a las caídas}

El número de trabajos analizados con relación al equilibrio y el miedo a la caída fue de 32 (26 EAC y 6 ENC). De todos ellos, 28 encontraron que el TCC producía mejoras significativas respecto al equilibrio, 2 respecto al miedo a las caídas y 4 en ambos aspectos, mientras que 3 no encontraron evidencias, aunque tampoco hallaron lo contrario. Según estos estudios, el TCC produjo mejoras significativas en sus practicantes en el equilibrio estático y dinámico, en los sistemas propioceptivo, vestibular y visual, y en el miedo a las caídas. En la tabla 3 se muestra un resumen de los resultados. 
Jiménez-Martín, P.J.; Meléndez-Ortega, A.; Albers, U.; López-Díaz, A. (2013). Beneficios del Tai Chi Chuan en la osteoartritis, el equilibrio y la calidad de vida. RICYDE. Rev. int. cienc. deporte. 32(9), 181-199. http://dx.doi.org/10.5232/ricyde2013.03206

Tabla 3.- Resultados de los estudios con relación a los beneficios del TCC sobre el equilibrio

\begin{tabular}{|c|c|c|c|c|c|c|c|}
\hline Estudios & $\begin{array}{c}\text { Equilibro } \\
\text { Estático }\end{array}$ & $\begin{array}{l}\text { Equilibrio } \\
\text { Dinámico }\end{array}$ & $\begin{array}{l}\text { Test10/50 m } \\
\text { caminando }\end{array}$ & Propioc & Vestib & Visual & $\begin{array}{l}\text { Miedo } \\
\text { Caída }\end{array}$ \\
\hline Audette y col. 2006 & $(\mathrm{p}<0.05)$ & - & - & - & - & - & - \\
\hline Barnett y col. (2003). & $(\mathrm{p}<0.01)$ & - & MNS & - & - & - & MNS \\
\hline Deschamps y col. (2009) & - & - & - & - & - & - & $(\mathrm{p}=0.005)$ \\
\hline Ding-Hai y Hui-Xin (2012) & $(\mathrm{p}<0.05)$ & $(\mathrm{p}<0.05)$ & - & - & - & - & - \\
\hline Fong y Ng (2006) & $(\mathrm{p}<0.001)$ & - & - & $(\mathrm{p}=0.001)$ & - & - & - \\
\hline Gatts y Woollacott (2007) & - & $(\mathrm{p} \leq 0.017)$ & - & - & - & - & - \\
\hline Gyllensten y col. (2010) & $(\mathrm{p}<0.005)$ & $(\mathrm{p}<0.01)$ & - & $(p=0.001)$ & - & - & - \\
\hline Hackney y Earhart (2008) & MNS & $(p=0.001)$ & $(p=0.046)$ & - & - & - & - \\
\hline Hain y col. (1999) & - & $(\mathrm{p}<0.001)$ & - & - & - & - & - \\
\hline Hall y col. (2009b) & NM & NM & - & - & - & - & NM \\
\hline Hartman y col. (2000) & MNS & - & MNS & - & - & - & - \\
\hline Lelard y col. (2010) & $\operatorname{MS}(¿ ?)$ & - & $\operatorname{MS}(i ?)$ & - & - & - & - \\
\hline Li y col. (2004) & $(\mathrm{p}<0.001)$ & - & $(p<0.001)$ & - & - & - & - \\
\hline Li y col. (2012) & $(\mathrm{p}<0.001)$ & $(\mathrm{p}<0.001)$ & $(\mathrm{p}<0.001)$ & - & - & - & - \\
\hline Mark y Ling (2003) & $(\mathrm{p}<0.05)$ & $(\mathrm{p}<0.05)$ & $(\mathrm{p}<0.05)$ & - & - & - & - \\
\hline McGibbon y col. 2005 & NM & $\operatorname{MS}(i ?)$ & $(\mathrm{p}=0.009)$ & $\operatorname{MS}(i ?)$ & $\operatorname{MS}(i ?)$ & - & - \\
\hline Qin y col. (2005) & $(\mathrm{p}<0.01)$ & - & - & - & - & - & - \\
\hline Ramachandran y col. (2007) & - & $(\mathrm{p}<0.001)$ & - & - & - & - & - \\
\hline Soto y col. (2008) & $\operatorname{MS}(i ?)$ & $\operatorname{MS}(i ?)$ & - & - & - & - & - \\
\hline Taggart (2002) & $(\mathrm{p}<0.001)$ & - & $(\mathrm{p}<0.05)$ & - & - & - & $(\mathrm{p}<0.001)$ \\
\hline Taylor y Coull (2012) & $\operatorname{MS}(i ?)$ & $\operatorname{MS}(i ?)$ & $\operatorname{MS}(i ?)$ & - & - & - & - \\
\hline Thorton y col. (2004) & - & $(\mathrm{p}<0.001)$ & - & - & - & - & - \\
\hline Tsang y col. (2004) & - & - & - & NMS & $(p=0.033)$ & $(\mathrm{p}=0.049)$ & - \\
\hline Tsang y Hui-Chan (2006) & - & - & - & - & $(p=0.016)$ & - & - \\
\hline Venglar (2005) & - & $\operatorname{MS}(i ?)$ & - & - & - & - & - \\
\hline Wolf y col. (1996) & - & - & - & - & - & - & $(\mathrm{p}=0.046)$ \\
\hline Wong y col. (2001) & $(\mathrm{p}<0.05)$ & $(\mathrm{p}<0.05)$ & - & $(\mathrm{p}<0.05)$ & $(\mathrm{p}<0.05)$ & $(\mathrm{p}<0.05)$ & - \\
\hline Woo y col. (2007) & NM & NM & NM & NM & NM & NM & - \\
\hline Wu y col. (2010) & $(\mathrm{p}<0.05)$ & - & - & - & - & - & $(\mathrm{p}<0.05)$ \\
\hline Xu, Hong y Chan 2004 & - & - & - & $(p=0.001)$ & - & - & - \\
\hline Yan (1998) & - & $(\mathrm{p}<0.001)$ & - & - & - & - & - \\
\hline Zhang y col. (2006) & $(\mathrm{p}<0.001)$ & - & $(p=0.404)$ & - & - & - & $(p=0.006)$ \\
\hline Total & $21(100 \%)$ & $17(100 \%)$ & $12(100 \%)$ & $7(100 \%)$ & $5(100 \%)$ & $3(100 \%)$ & $7(100 \%)$ \\
\hline Total MS & $16(76.1 \%)$ & $15(88.2 \%)$ & $9(75 \%)$ & $5(71.4 \%)$ & $4(80 \%)$ & $2(66.6 \%)$ & $5(71.4 \%)$ \\
\hline
\end{tabular}

Nota: $M S=$ Mejora significativa, $M N S=$ Mejora no significativa; $N S=$ No mejora; $i ?=$ Sin especificar 
Jiménez-Martín, P.J.; Meléndez-Ortega, A.; Albers, U.; López-Díaz, A. (2013). Beneficios del Tai Chi Chuan en la osteoartritis, el equilibrio y la calidad de vida. RICYDE. Rev. int. cienc. deporte. 32(9), 181-199. http://dx.doi.org/10.5232/ricyde2013.03206

\section{Calidad de Vida}

El número de trabajos analizados con relación a la calidad de vida fue de 30 (18 EAC y 12 ENC). Según estos estudios, el TCC produjo mejoras significativas en sus practicantes en la calidad de vida en las dimensiones del bienestar emocional (en particular sobre el estado de ánimo en general, estrés, ansiedad, depresión y enfado-tensión), la percepción de sí mismo (autoeficacia y miedo a las caídas) y en el bienestar corporal (trastornos de sueño). En las tablas 4 y 5 se muestra un resumen de los resultados.

Tabla 4.- Resultados de los estudios con relación a la Calidad de Vida: Bienestar emocional

\begin{tabular}{|c|c|c|c|c|c|c|}
\hline \multirow[b]{2}{*}{ Estudios } & \multicolumn{6}{|c|}{ Bienestar Emocional } \\
\hline & Ánimo & Depresión & $\begin{array}{l}\text { Enfado- } \\
\text { Tensión }\end{array}$ & Ansiedad & Estrés & $\begin{array}{c}\text { Desórdenes } \\
\text { Emocionales }\end{array}$ \\
\hline Barnett y col. (2003) & - & - & - & - & - & - \\
\hline Baron y Faubert (2005) & NS & - & - & NS & - & - \\
\hline Blake y Batson (2009) & $(\mathrm{p}=0.002)$ & - & - & - & - & - \\
\hline Brown y col. (1995) & $(\mathrm{p}<0.006)$ & $(\mathrm{p}<0.05)$ & $(\mathrm{p}<0.008)$ & MNS & MNS & MNS \\
\hline Chen y col. (2008) & - & - & - & - & - & - \\
\hline Deschamps y col. (2009) & - & $(\mathrm{p}=0.005)$ & - & - & & - \\
\hline Field y col. (2010) & - & - & - & $(\mathrm{p}=0.02)$ & - & - \\
\hline Fransen y col. (2007) & - & NM & - & NM & $\mathrm{NM}$ & - \\
\hline Galantino y col. (2005) & $(\mathrm{p}=0.005)$ & - & $(p=0.005)$ & $(\mathrm{p}=0.001)$ & - & - \\
\hline Hall y col. (2009b) & - & - & - & - & - & - \\
\hline Hernádez-Reif y col. (2001) & - & - & - & $(\mathrm{p}=0.001)$ & & $(\mathrm{p}=0.001)$ \\
\hline Hoffmann-Smith y col. (2009) & $\operatorname{MS}(p=i ?)$ & $\operatorname{MS}(p=i ?)$ & $\operatorname{MS}(p=i ?)$ & $\operatorname{MS}(\mathrm{p}=i ?)$ & & - \\
\hline Irwin y col. (2008) & - & $(\mathrm{p}<0.001)$ & - & - & - & - \\
\hline Jin (1989) & $(\mathrm{p}<0.001)$ & $(\mathrm{p}<0.001)$ & $(\mathrm{p}<0.001)$ & $(\mathrm{p}<0.001)$ & $(\mathrm{p}<0.001)$ & - \\
\hline Jin (1992) & $(\mathrm{p}<0.001)$ & - & $\operatorname{MS}(p=i ?)$ & - & - & $\operatorname{MS}(p=i ?)$ \\
\hline Lee y col. (2009) & - & - & - & - & - & - \\
\hline Li y col. (2001) & - & - & - & - & & - \\
\hline Li y col. (2004) & - & - & - & - & - & - \\
\hline Liu y col. (2008) & - & $(\mathrm{p}<0.05)$ & - & - & $(\mathrm{p}<0.05)$ & MNS \\
\hline Taggart (2002) & - & - & - & - & - & - \\
\hline Taylor y Froelicher (2004) & - & - & - & - & - & - \\
\hline Taylor y col. (2006) & $(\mathrm{p}=0.001)$ & - & $(\mathrm{p}=0.002)$ & $(\mathrm{p}=0.002)$ & $(\mathrm{p}=0.009)$ & - \\
\hline Toda y col. (2011) & $(\mathrm{p}=0.05)$ & $(\mathrm{p}<0.05)$ & $(\mathrm{p}=0.001)$ & MNS & - & - \\
\hline Robins y col. (2006) & - & - & - & - & $(\mathrm{p}=0.165)$ & $\operatorname{MS}(p=i ?)$ \\
\hline Reid-Arndt y col. (2011) & MNS & MNS & NS & - & $(\mathrm{p}<0.05)$ & - \\
\hline Song y col. (2007) & - & - & - & - & $(\mathrm{p}=0.005)$ & MNS \\
\hline Wolf y col. (1996) & - & - & - & - & - & - \\
\hline Wu y col. (2010) & - & - & - & - & - & - \\
\hline Yeh y col. (2008). & - & - & - & - & - & - \\
\hline Zhang y col. (2006) & - & - & - & - & - & - \\
\hline Total que analizan & $10(100 \%)$ & $9(100 \%)$ & $8(100 \%)$ & $10(100 \%)$ & $8(100 \%)$ & $6(100 \%)$ \\
\hline Total mejora significativa & $8(80.0 \%)$ & $7(77.7 \%)$ & $7(87.5 \%)$ & $6(60.0 \%)$ & $6(75.0 \%)$ & $3(50.0 \%)$ \\
\hline
\end{tabular}

Nota: $\mathrm{MS}=$ Mejora significativa, $\mathrm{MNS}=$ Mejora no significativa; $\mathrm{NS}=$ No mejora; $\underset{\iota}{ }=\mathrm{S}$ Sin especificar 
Jiménez-Martín, P.J.; Meléndez-Ortega, A.; Albers, U.; López-Díaz, A. (2013). Beneficios del Tai Chi Chuan en la osteoartritis, el equilibrio y la calidad de vida. RICYDE. Rev. int. cienc. deporte. 32(9), 181-199. http://dx.doi.org/10.5232/ricyde2013.03206

Tabla 5.- Resultados Calidad de Vida: Percepción de sí mismo y Bienestar Corporal

\begin{tabular}{|c|c|c|c|c|}
\hline \multirow{2}{*}{ Estudios } & \multicolumn{2}{|c|}{ Percepción de sí mismo } & \multicolumn{2}{|c|}{ Bienestar Corporal } \\
\hline & Auto-eficacia & Autoestima & Miedo Caída & Trastornos de sueño \\
\hline Barnett y col. (2003) & - & - & MNS & - \\
\hline Baron y Faubert (2005) & - & - & - & - \\
\hline Blake y Batson (2009) & - & $(\mathrm{p}=0.01)$ & - & - \\
\hline Brown y col. (1995) & - & MNS & - & - \\
\hline Chen y col. (2008) & - & - & - & - \\
\hline Deschamps y col. (2009) & $(p=0.003)$ & - & $(\mathrm{p}<0.001)$ & - \\
\hline Field y col. (2010) & - & - & - & - \\
\hline Fransen y col. (2007) & MNS & - & - & - \\
\hline Galantino y col. (2005) & - & - & - & - \\
\hline Hall y col. (2009b) & - & - & NM & - \\
\hline Hernádez-Reif y col. (2001) & - & - & - & $(\mathrm{p}=0.001)$ \\
\hline Hoffmann-Smith y col. (2009) & - & - & - & $\operatorname{MS}(p=i ?)$ \\
\hline Irwin y col. (2008) & - & - & - & $(\mathrm{p}=0.05)$ \\
\hline $\operatorname{Jin}(1989)$ & - & - & - & - \\
\hline $\operatorname{Jin}(1992)$ & - & - & - & - \\
\hline Lee y col. (2009) & $(p<0.05)$ & - & - & - \\
\hline Li y col. (2001) & $\operatorname{MS}(p=i ?)$ & - & - & - \\
\hline Li y col. (2004) & - & - & - & $(\mathrm{p}=0.001)$ \\
\hline Liu y col. (2008) & - & - & - & - \\
\hline Taggart (2002) & - & - & $(p<0.001)$ & - \\
\hline Taylor y Froelicher (2004) & $(\mathrm{p}=0.001)$ & - & - & - \\
\hline Taylor y col. (2006) & $(\mathrm{p}=0.001)$ & - & - & - \\
\hline Toda y col. (2011) & - & - & - & - \\
\hline Robins y col. (2006) & - & - & - & - \\
\hline Reid-Arndt (2011) & - & - & - & - \\
\hline Song y col. (2007) & MNS & - & - & - \\
\hline Wolf y col. (1996) & - & - & $(\mathrm{p}=0.046)$ & - \\
\hline Wu y col. (2010) & - & - & $(\mathrm{p}<0.05)$ & - \\
\hline Yeh y col. (2008). & - & - & - & $(\mathrm{p}=0.04)$ \\
\hline Zhang y col. (2006) & - & - & $(\mathrm{p}=0.006)$ & - \\
\hline Total que analizan & $7(100 \%)$ & $2(100 \%)$ & $7(100 \%)$ & $5(100 \%)$ \\
\hline Total mejora significativa & $5(71.4 \%)$ & $1(50.0 \%)$ & $5(71.4 \%)$ & $5(100 \%)$ \\
\hline
\end{tabular}

Nota: $\mathrm{MS}=$ Mejora significativa, $\mathrm{MNS}=$ Mejora no significativa; $\mathrm{NS}=$ No mejora; $₫ ?=$ Sin especificar. 
Jiménez-Martín, P.J.; Meléndez-Ortega, A.; Albers, U.; López-Díaz, A. (2013). Beneficios del Tai Chi Chuan en la osteoartritis, el equilibrio y la calidad de vida. RICYDE. Rev. int. cienc. deporte. 32(9), 181-199. http://dx.doi.org/10.5232/ricyde2013.03206

\section{Discusión}

Este apartado se ha estructurado en el mismo orden que la presentación de resultados.

\section{Diseño de los estudios analizados}

En esta revisión se ha podido comprobar que el estilo Yang de TCC en sus formas simplificadas modernas es el más utilizado en las intervenciones. Este aspecto coincide con todas las revisiones sistemáticas encontradas (Dechamps, Lafont y Bourdel-Marchasson, 2007; Hall y col., 2009a; Hong y Li, 2007; Lee, Lam y Ernst, 2008a; Lee, Pittler y Ernst, 2008b; Li y col., 2009b; Logghe, Verhagen, Rademaker, Bierma-Zeinstra, van Rossum, Faber, y Koes, 2010; Low y col., 2009; Rogers y col., 2009; Wang y col., 2010; Wang y col., 2004 y Wayne, Kiel, Krebs, Davis, Savetsky-German, Connelly, y Buring, 2007). El estilo Yang es el más extendido y practicado a nivel mundial (Low y col., 2009), y es el que mejor se adecua, por sus características de ejecución, a los ancianos, perfil de población en el que se centran la mayor parte de los estudios (Dechamps y col., 2007).

Los beneficios que aporta el TCC son similares independientemente de que se esté interviniendo con una forma tradicional o moderna, sin embargo, dado que existen diferentes estilos y formas, con una gran variación en cuanto al número de movimientos, tiempos de ejecución, número y tipo de posturas, estamos de acuerdo con otros autores (Dechamps y col., 2007; Hall y col., 2009a; Lee et al., 2008b; Li y col., 2009b; Logghe y col., 2010; Rogers y col., 2009; Wang y col., 2010; Wayne y col. 2007) en que esto supone todavía un problema importante a la hora de generalizar resultados.

Respecto al tiempo de práctica necesario para comenzar a registrar los beneficios del TCC estamos de acuerdo con Brown, Wang, Ward, Ebbeling, Fortlage, Puleo, Benson, y Rippe (1995); Chang y col. (2010) y Wang y col. (2010) cuando hablan de la necesidad de estandarizar la duración y frecuencia de práctica para poder comparar resultados, y con Spirduso y col. (2005), cuando señalan que sería interesante realizar estudios longitudinales para determinar cómo es la evolución de los efectos en el tiempo; en investigar los efectos del TCC en una simple sesión; así como en la necesidad de establecer comparaciones respecto a otras actividades físicas, que se están recomendando a persona mayores en cuanto al tiempo en que comienza a aportar beneficios.

Respecto a las características muestrales, este trabajo vuelve a confirmar, como en el resto de trabajos de revisión sistemática encontrados, el escaso número de participantes, un perfil mayoritario de personas mayores y la no diferenciación respecto al género. Dechamps y col. (2007) incidan que el TCC se ha asociado con la población adulta-anciana ( $>55$ años), descuidándose el estudio de sus efectos sobre otros rangos de edad, y estos mismos autores junto con Jackson, Wluka, Teichtahl, Morris, y Cicuttini (2004) y Song, Lee, Lam, y Bae (2003) señalan que los efectos de esta actividad son diferentes entre hombres y mujeres cuando se están midiendo efectos psicológicos o sobre determinadas patologías como la OA.

Por último, señalar que los resultados obtenidos en este trabajo deben ser considerados con cierta prudencia, dadas las deficiencias y diferencias metodológicas registradas en los estudios. Este punto es el común denominador que aparece destacado en todos los trabajos de revisión sistemática y meta-análisis encontrados: muestras reducidas, carencias en los criterios de inclusión y exclusión de los participantes, deficiencias en los análisis estadísticos, carencias en la estandarización de la medición de resultados, etc. (Hall y col., 2009a; Hong y Li, 2007; 2008; Lee et al. 2008b; Li y col. 2009b, Logghe y col., 2010; Low y col., 2009; Wang y col., 2004; Wang y col. 2010; Wayne y col., 2007; Yeh y col., 2009). Sin embargo, también hay que señalar que en estas revisiones los instrumentos de evaluación utilizados 
Jiménez-Martín, P.J.; Meléndez-Ortega, A.; Albers, U.; López-Díaz, A. (2013). Beneficios del Tai Chi Chuan en la osteoartritis, el equilibrio y la calidad de vida. RICYDE. Rev. int. cienc. deporte. 32(9), 181-199. http://dx.doi.org/10.5232/ricyde2013.03206

(CONSORT, Jadad Score, PEDro rating scale, NICE, Delphi criteria list y ABC) centran su atención exclusiva en el análisis del diseño metodológico de la investigación, y no abordan otros aspectos fundamentales con relación a la propia práctica del TCC como: el estilo practicado, la dosificación, la experiencia de los instructores, la altura en las posturas, el tiempo de ejecución de cada forma, o la experiencia de los instructores.

\section{Osteoartritis}

La OA es una de las afecciones crónicas que más afectan a personas mayores de 65 años, en especial a las mujeres, y donde la obesidad juega un importante factor de riesgo que agrava la situación (Bennel y Hinman 2010; Jackson y col., 2004). Aunque en los estudios localizados en este trabajo este perfil es el más representativo, respecto a la obesidad no se adoptan medidas de control en las intervenciones.

En cuanto al diagnóstico de partida, 5 estudios aportan datos sobre el tiempo que se padece esta patología y 6 ofrecen información sobre el grado de OA de los participantes, sin embargo, a nivel radiológico, sólo hay 2 estudios (Song y col., 2003 y Lee y col., 2009) que utilicen esta técnica, y no incluyen datos sobre el plano de visión lateral-axial y rodilla flexionada como recomiendan Vignon y col. (2006) para un correcto diagnóstico de la OA. Ninguno aporta tampoco información sobre la localización de la OA en la rodilla, es decir, si es patelofemoral o en el compartimento tibiofemoral medial o lateral, cuando ésta guarda una estrecha relación con las estrategias de compensación postural frente al dolor como se está evidenciando desde los estudios biomecánicos (Englund, 2010 y Jackson y col., 2004). Tampoco utilizan como referencia, para la toma de datos o la exposición de resultados, la clasificación, distinción y evolución de los pacientes según el tiempo, grado y ubicación de la OA de partida.

En los estudios analizados en este trabajo, el TCC produjo mejoras significativas en sus practicantes en la percepción de la funcionalidad física y la rigidez, mientras que en el dolor hay tanto evidencias a favor como en contra. Estos resultados coinciden en cuanto al dolor con los estudios de revisión sistemática realizados por Lee et al. (2008b), Hall y col. (2009a) y Kang, Lee, Posadzki y Ernst, (2011) pero no respecto a la mejora de la percepción de la funcionalidad física. Sin embargo, los autores de estos estudios señalan la existencia de una tendencia positiva del TCC en la mejora del control del dolor y la funcionalidad física que debería tenerse en cuenta por la reducción de costes médicos que podría suponer. Los beneficios encontrados sobre la rigidez articular en este trabajo no se pueden comparar porque no es un factor tratado en estas revisiones, y respecto a la no mejora en el rango del movimiento, destacar que sólo hay un artículo encontrado que haga referencia a este aspecto (Song y col. 2003) e indica que, dada la reducida amplitud de movimiento que tiene el TCC, y más en ancianos, este factor es difícilmente afectable por esta actividad.

\section{Equilibrio y el miedo a la caída}

Los estudios analizados con relación al equilibrio se pueden clasificar en dos grupos: 1) específicos, sobre la influencia del TCC en los sistemas propioceptivo, vestibular y visual (Fong y Ng, 2006; Gyllensten, Hui-Chan, y Tsang, 2010; Li y col. 2009a; McGibbon, Krebs, Parker, Scarborough, Wayne, y Wolf, 2009; Tsang, Wong, Fu, y Hui-Chan, 2004; Tsang y Hui-Chan, 2006; Wong, Lin, Chou, Tang, y Wong, 2001; Woo, Hong, Lau, y Lynn, 2007; $\mathrm{Xu}$, Hong y Chan, 2004); y 2) generales, que analizan este factor de modo secundario, junto a otros parámetros que afectan a la salud sin profundizar en los aspectos sensorio-motores del equilibrio. 
Jiménez-Martín, P.J.; Meléndez-Ortega, A.; Albers, U.; López-Díaz, A. (2013). Beneficios del Tai Chi Chuan en la osteoartritis, el equilibrio y la calidad de vida. RICYDE. Rev. int. cienc. deporte. 32(9), 181-199. http://dx.doi.org/10.5232/ricyde2013.03206

Propiocepción. El TCC, al realizarse de forma repetitiva con movimientos lentos y continuos en el espacio, demandaría una gran conciencia corporal del practicante respecto a la aceleración, fuerza, trayectoria y ejecución de los movimientos, favoreciendo una mejor representación cortical de las articulaciones y sensación de posición (Ramachandran, Rosengren, Yang, y Hsiao-Wecksler, 2007; Fong y Ng, 2006; Wayne, Krebs, Wolf, GillBody, Scarborough, McGibbon, Kaptchuk, y Parker, 2004). De los estudios analizados Fong y Ng (2006), Gyllensten y col. (2010), Wong y col. (2001) y Xu y col. (2004) confirman que el TCC produce una mejora en el sistema propioceptivo de sus practicantes, mientras que Tsang y col. (2004) y Woo y col. (2007) no encontraron ninguna mejora significativa, si bien la causa puede estar en los instrumentos de medición utilizados y el tiempo reducido de práctica.

En los estudios sobre la propiocepción destacan dos aspectos: 1) parece ser que la mejora de este parámetro está muy relacionado con un mínimo de tiempo de práctica (Fong y Ng, 2006; Li y col. 2009b; Wong y col. 2001; Xu y col., 2004) y 2) que las mejoras en el equilibrio tiene especial relación con la mejora en la propiocepción del tobillo y la reducción de la dependencia de los movimientos compensatorios de la cadera, beneficio que parece ser específico a la práctica de esta actividad (McGibbon y col. 2009; Xu y col., 2004).

Sistema vestibular. De los estudios analizados McGibbon y col. (2009), Tsang y col. (2004), Tsang y Hui-Chan (2006) y Wong y col. (2001) confirman que la práctica del TCC produce una mejora en la utilización del sistema vestibular para el control del equilibrio, mientras que Woo y col. (2007) no encontraron ninguna mejora significativa. Hay que ser prudentes con estos resultados porque, al igual que señalan Wayne y col. (2004), en la práctica totalidad de estudios analizados no se incluyen muestras con pacientes afectados por problemas vestibulares específicos. Las causas que producen esta mejora se asocian a los movimientos de rotación de cabeza y tronco que toman lugar junto al cambio de peso en los desplazamientos del TCC y el seguimiento con la mirada del movimiento de las manos (Wayne y col. 2004). El tiempo de práctica también es un factor clave en este sistema (Tsang y col., 2004; Tsang y Hui-Chan, 2006; Wong y col., 2001), un aspecto, que por otro lado, y tal y como señalan Low y col. (2009), puede ser un problema en ancianos donde la adherencia a la práctica presenta ciertas dificultades.

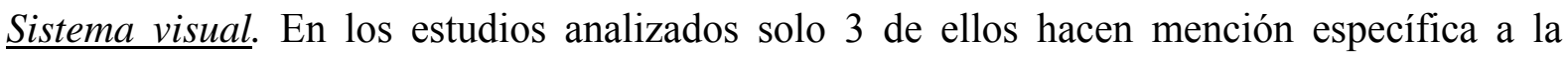
medición de este parámetro y de ellos, dos (Tsang y col., 2004 y Wong y col., 2001) confirman una mejora significativa respecto al grado de oscilación corporal que se produce en los ancianos cuando son sometidos a una prueba de equilibrio sobre una plataforma de referencia oscilante con ojos abiertos y cerrados; y uno no (Woo y col., 2007).

Equilibrio estático y dinámico. A pesar de las mejoras significativas registradas en este trabajo respecto a la dimensión estática (16 estudios de 21) y dinámica (15 estudios de 17) del equilibrio, se debe señalar, que en estas investigaciones se aborda este aspecto desde una visión general aplicando test estandarizados (Single-Leg Stance, Berg Balance Scale, Functional Reach Test, Romberg Test, 6-8-10 or 50m foot walking speed test) sin llegar a profundizar en las causas que han dado lugar a dicha mejora.

Caídas. En los estudios se analizan dos aspectos: la confianza frente al miedo a la caída y la reducción en el número de caídas. En ambos, aunque los estudios específicos analizados en este trabajo confirmen que hay una mejora significativa, en las revisiones sistemáticas realizadas por Rogers y col. (2009) y Verhagen, Immink, Meulen, y Bierma-Zeinstra (2004) se encontraron investigaciones en los que no se confirmaban mejoras respecto al grupo de control en ambos parámetros, por lo que hay que seguir siendo prudentes en este aspecto. 
Jiménez-Martín, P.J.; Meléndez-Ortega, A.; Albers, U.; López-Díaz, A. (2013). Beneficios del Tai Chi Chuan en la osteoartritis, el equilibrio y la calidad de vida. RICYDE. Rev. int. cienc. deporte. 32(9), 181-199. http://dx.doi.org/10.5232/ricyde2013.03206

El número de ensayos aleatorios controlados analizados en este trabajo (26 de 32) es superior al resto de revisiones sistemáticas específicas encontradas sobre esta temática: Logghe y col. (2010) (15 EAC y 6 ENC), Low y col. (2009) (7 EAC), Verhagen y col. (2004) (4 EAC y 5 ENC), Wang y col. (2004) (2 EAC y 9 ENC) y Wayne y col. (2004) (10 EAC y 14 ENC). Además, todas las mejoras significativas registradas en este trabajo coinciden con las encontradas en la revisión de Wayne y col. (2004); en la mejora del equilibrio en general y la confianza respecto a la caídas con Wang y col. (2004); en la confianza frente a la caída con Low y col. (2009) y Logghe y col. (2010), pero no coincide con los resultados encontrados por Logghe y col. (2010) respecto a que se produzcan mejoras sobre el equilibrio estático y dinámico o con Verhagen y col. (2004) respecto al riesgo de caídas.

En todas estas revisiones se pone de manifiesto que las causas que conducen a estas mejoras todavía no son claras, sin embargo, se puede destacar que desde el ámbito de la biomecánica se están ofreciendo importantes respuestas, a partir de los estudios que se están realizando respecto al TCC y la ejecución de la marcha, patrones de movimiento en la superación de obstáculos, distribución del peso en la planta de los pies y traslación del centro de gravedad (Englund, 2010; Hong y Li, 2007; Jackson y col., 2004; Ramachandran y col., 2007; Tsang y col., 2004; Wu, 2008). Aquí se considera que las mejoras guardan una estrecha relación con el cambio constante de peso en el tren inferior que hay en la forma, y el tiempo de estancia sobre una sola pierna. Estos estudios parecen confirmar mejoras significativas en la amplitud de zancada (Gatts y Woollacot, 2007; Gyllensten y col., 2010; Mark y Ling, 2003), mejor control direccional (Gyllensten y col., 2010) y mayor habilidad para superar obstáculos (Gyllensten y col., 2010; Ramachandran y col. 2007).

\section{Calidad de vida}

El análisis de los efectos psicológicos que produce el TCC sobre sus practicantes se podría centrar en dos ámbitos: la función cognitiva y la calidad de vida. Respecto al primero, el número de intervenciones realizadas es muy reducido (Chang y col. 2010), mientras que el segundo, aunque contiene la mayor parte de los estudios realizados, presenta una gran confusión conceptual dadas las múltiples y diferentes formas con las que son clasificadas las variables que constituyen la calidad de vida por los distintos autores. Para solventar esta problemática, en este trabajo de revisión se ha tomado como referencia el modelo de Spidurso, Francis y MacRae (2005), en el que se definen y jerarquizan los parámetros que engloba la calidad de vida.

Según los resultados, el TCC produce mejoras significativas en la calidad de vida de sus practicantes en las dimensiones del bienestar emocional (en particular sobre el estado de ánimo en general, estrés, ansiedad, depresión y enfado-tensión), la percepción de sí mismo (autoeficacia y miedo a las caídas) y el bienestar corporal (trastornos de sueño). Estos resultados coinciden con los encontrados en las revisiones sistemáticas realizadas por Dechamps y col., 2007) Hall y col. (2009a), Lee y col. (2008b), Rogers y col. (2009), Wang y col. (2010) y Wang y col. (2004), excepto en la mejora de la autoestima, ya que en este trabajo el número de estudios encontrados sobre esta temática es mínimo.

Las causas que producen estos efectos positivos todavía no están claras. Algunas de las explicaciones que se ofrecen son: la relajación que constantemente se persigue en el TCC y el trabajo respiratorio que lo acompaña (Irwin, Olmstead y Motivala, 2008; Li, Fisher, Harmer, Irbe, Tearse, y Weimer, 2004; Toda, Den, Hasegawa-Ohira, y Morimoto, 2011; y Taylor, Haskell, y Waters, 2006); las expectativas positivas de los participantes que asocian esta actividad con salud y calma mental generando el síndrome de auto profecía cumplida (Brown y col., 1995 y Jin, 1989, 1992); el carisma del profesorado que imparte las clases 
Jiménez-Martín, P.J.; Meléndez-Ortega, A.; Albers, U.; López-Díaz, A. (2013). Beneficios del Tai Chi Chuan en la osteoartritis, el equilibrio y la calidad de vida. RICYDE. Rev. int. cienc. deporte. 32(9), 181-199. http://dx.doi.org/10.5232/ricyde2013.03206

(Deschamps, Onifade, Decamps, y Bourdel-Marchasson, 2009; Li, McAuley, Harmer, Duncan, y Cahumeton, 2001; Taylor y col., 2006 y Robins, McCain, Gray, Elswick, Walter, y McDade, 2006); o el olvido de los problemas que causan ansiedad por estar inmerso en una actividad (Jin, 1989).

Respecto al factor auto-eficacia, de la dimensión percepción de uno mismo, los estudios se centran en algunos autores (Deschamps y col. 2009, Li y col. 2001 y Taylor y col. 2006) en el problema de la adherencia a los programas, investigando las barreras psicológicas y sociales que encuentran las personas mayores hacia el ejercicio físico. En general, los estudios confirman mejoras en este factor aunque la relación de estos efectos con la adherencia todavía no está clara. Respecto al factor de mejora en los trastornos del sueño incluidas en la dimensión bienestar corporal, los estudios analizados (Li y col., 2004; Irwin y col., 2008; Yeh, Mietus, Peng, Phillips, Davis, Wayne, Goldberger, y Thomas, 2008) confirman mejoras significativas en el sueño nocturno y diurno, si bien es verdad que Irwin y col. (2008) encontraron que estas mejoras se manifiestan cuando las personas parten de una mala calidad de sueño y que es necesario un tiempo mínimo de práctica.

Por otro lado, también es importante resaltar que todos los efectos positivos encontrados respecto a las diferentes dimensiones de la calidad de vida en este trabajo, parecen coincidir con los que se están obteniendo en otros estudios sobre actividad física y deporte, según describen Aparicio, Carbonell-Baeza y Delgado-Fernández (2010), Martínez, Santos y Casimiro (2009) y Spirduso y col. (2005). Esto indica que esta actividad aporta los mismos beneficios psicológicos que otras actividades físicas. Sin embargo, coincidimos con Deschamps y col. (2009) y Yan, (1998), en que aunque esta actividad muestre los mismos beneficios psicológicos que otro tipo de ejercicios, los beneficios paralelos que aporta sobre otros aspectos de la salud: incremento de la fuerza muscular en el tren inferior, mejoras de los parámetros cardiovasculares y respiratorios, fortalecimiento del sistema inmunológico, etc...(Rogers, Larkey y Keller, 2009) y las condiciones que acompañan a su práctica (carácter no competitivo y tranquilo, la no necesidad de un equipamiento específico, tiempo y lugar de práctica muy flexible) la convierten en una actividad física muy recomendable para los ancianos.

\section{Conclusiones}

Los efectos del TCC sobre la osteoartritis de rodilla son contradictorios, aunque se está registrando una tendencia positiva respecto al control del dolor y las mejoras en la funcionalidad física.

El TCC produce mejoras significativas en sus practicantes en el equilibrio estático y dinámico y en la confianza de la persona respecto al miedo a la caída. Las mejoras del sistema propioceptivo tiene una gran relación con las mejoras en la propiocepción en el tobillo, y tanto en este sistema como en el vestibular, los beneficios están relacionados con un mínimo de práctica en el tiempo.

A nivel psicológico la práctica del TCC mejora la calidad de vida de sus practicantes en las dimensiones del bienestar emocional (estado de ánimo en general, estrés, ansiedad, depresión y enfado-tensión), la percepción de sí mismo (autoeficacia y miedo a las caídas) y el bienestar corporal (trastornos de sueño). Sin embargo, estas mejoras son equivalentes a las que se consiguen con otras actividades de baja y mediana intensidad. En cuanto a los estudios sobre los beneficios del TCC en la funcionalidad cognitiva todavía son muy reducidos. Sin embargo, esta línea contiene un gran potencial en el trabajo con ancianos, dada la importancia 
Jiménez-Martín, P.J.; Meléndez-Ortega, A.; Albers, U.; López-Díaz, A. (2013). Beneficios del Tai Chi Chuan en la osteoartritis, el equilibrio y la calidad de vida. RICYDE. Rev. int. cienc. deporte. 32(9), 181-199. http://dx.doi.org/10.5232/ricyde2013.03206

que tiene a la hora de facilitar su inclusión en los programas de intervención y la recogida de datos.

Los resultados obtenidos deben tomarse con cierta cautela debido a que un gran número de los estudios analizados muestran deficiencias metodológicas en el diseño de investigación. Queda también por definir un marco homogéneo de intervención específica con el TCC que facilite la comparación de resultados entre los diferentes estudios según: los estilos y formas de TCC utilizados, dosificación, metodología de enseñanza e influencia del profesorado, y realizar mayor número de estudios específicos a nivel biomecánico y físiológico para determinar las posibles causas que producen las mejoras registradas. Por último, incidir que muchos de los estudios analizados en este trabajo, afrontan la medición de los beneficios del TCC con pacientes que sufren diferentes patologías o enfermedades. Aunque el número de estudios encontrados para cada patología es muy reducido, los resultados positivos encontrados indican que esta línea ofrece un interesante potencial de investigación.

\section{Referencias bibliográficas}

Aparicio, V.A.; Carbonell-Baeza, A., y Delgado-Fernández, M. (2010). Beneficios de la Actividad Física en Personas Mayores. Revista Internacional de Medicina y Ciencias de la Actividad Física y el Deporte, 10(40), 556-576.

Audette, J. F.; Jin, Y.S.; Newcomer, R.; Stein, L.; Duncan, G., \& Frontera, W.R. (2006). Tai Chi versus brisk walking in elderly women. Age Ageing, 35(4), 388-393.

Barnett, A.; Smith, B.; Lord, S.; Williams, M., \& Baumand, A. (2003). Community-based group exercise improves balance and reduces falls in at-risk older people: a randomised controlled trial. Age Ageing, 32(4), 407-414.

Baron, L.J., \& Faubert, C. (2005). The role of Tai Chi Chuan in reducing state anxiety and enhancing mood of children with special needs. Journal of Bodywork and Movement Therapies, 9(2), 120-133.

Bennell, K.L., \& Hinman, R.S. (2001). A review of the clinical evidence for exercise in osteoarthritis of the hip and knee. J Sci Med Sport, 14, 4-9.

Blake, H., \& Batson, M. (2009). Exercise intervention in brain injury: a pilot randomized study of Tai Chi Qigong. Clin Rehabil, 23, 589-598.

Brismée, J.M.; Paige, R.L.; Chyu, M.C.; Boatright, J.D.; Hagar, J.M.; McCaleb, J.A.; Quintela, M.M.; Feng, D.; Xu, K.T., \& Shen, C.L. (2007). Group and home-based tai chi in elderly subjects with knee osteoarthritis: a randomized controlled trial. Clin Rehabil, 21 (2), 99-111.

Brown, D.R.; Wang, Y.; Ward, A.; Ebbeling, C.B.; Fortlage, L.; Puleo, E.; Benson, H., \& Rippe, J.M. (1995). Chronic psychological effects of exercise and exercise plus cognitive strategies. Med. Sci. Sports Exerc, 27(5), 765-775.

Chang, Y.K. ; Nien, Y.H. ; Tsai, C.L., \& Etnier, J. (2010). Physical Activity and Cognition in Older Adults: The Potential of Tai Chi Chuan. JAPA, 18, 451-472.

Chen, K.M. ; Chen, W.T., \& Huang, M-F. (2006). Development of the simplified Tai Chi exercise program (STEP) for frail older adults. Complementary Therapies in Medicine, $14,200-206$.

Chen, C. H.; Yen, M.; Fetzer, S.; Lo, L.H., \& Lam, P. (2008). The Effects of Tai Chi Exercise on Elders with Osteoarthritis: A Longitudinal Study. Asian Nursing Research, 2(4), 235-241. 
Jiménez-Martín, P.J.; Meléndez-Ortega, A.; Albers, U.; López-Díaz, A. (2013). Beneficios del Tai Chi Chuan en la osteoartritis, el equilibrio y la calidad de vida. RICYDE. Rev. int. cienc. deporte. 32(9), 181-199. http://dx.doi.org/10.5232/ricyde2013.03206

Dechamps, A.; Lafont, L., \& Bourdel-Marchasson, I. (2007). Effects of Tai Chi exercises on self-efficacy and psichological health. Eur Rev Aging Phys Act, 4, 25-32.

Deschamps, A.; Onifade, C.; Decamps, A., \& Bourdel-Marchasson, I. (2009). HealthRelated Quality of Life in Frail Institutionalized Elderly: Effects of a Cognition-Action intervention and Tai Chi. JAPA, 17(2), 236-248.

Ding-Hai, Y., \& Hui-Xin, Y. (2012). The effect of Tai Chi intervention on balance in older males. Journal of Sport and Health Science, 1, 57-60.

Englund, M. (2010). The role of biomechanics in the initiation and progression of OA of the Knee. Best Practice \& Research Clinical Rheumatology, 24, 39-46.

Field, T.; Diego, M., \& Hernández-Reif, M. (2010). Tai chi/yoga effects on anxiety, heartrate, EEG and math computations. Complementary Therapies in Clinical Practice, 16, 235-238.

Fransen, M.; Nairn, L.; Winstanley, J.; Lam, P., \& Edmonds, J. (2007). Physical Activity for Osteoarthritis Management: A Randomized Controlled Clinical Trial Evaluating Hydrotherapy or Tai Chi Classes. Arthritis \&Rheumatism, 57(15), 407414.

Fong, S.M., \& Ng, G.Y. (2006). The Effects on Sensoriomotor Performance and Balance with Tai Chi Training. Archives of Physical Medicine and Rehabilitation, 87(1), 82-87.

Galantino, M.L.; Shepard, K.; Krafft, L.; Laperriere, A.; Ducette, J.; Sorbello, A.; Barnish, M.; Condoluci, D., \& Farrar, J.T. (2005). The Effect of Group Aerobic Exercise and $T$ 'ai Chi on Functional Outcomes and Quality of Life for Persons Living with Acquired Immunodeficiency Syndrome. The Journal of Alternative and Complementary Medicine, 11(6), 1085-1092.

Gatts, S.K., \& Woollacott, S.H. (2007). How Tai Chi improves balance: Biomechanics of recovery to a walking slip in impaired seniors. Gait \& Posture, 25, 205-214.

Gyllensten, A.L.; Hui-Chan, C., \& Tsang, W. (2010). Stability Limits, Single-Leg Jump, and Body Awareness in Older Tai Chi Practitioners. Arch Phys Med Rehabil, 91, 215220.

Hackney, M., \& Earhart, G. (2008). Tai Chi improves balance and mobility in people with Parkinson disease. Gait \& Posture, 28, 456-460.

Hain, T.C.; Fuller, L.; Weil, L., \& Kotsias, J. (1999). Effects of T'ai Chi on Balance. Arch Otolaryngol Head Neck Surg, 125, 1191-1195.

Hall, A.; Maher, C.; Latimer, J., \& Ferreira, M. (2009s). The Effectiveness of Tai Chi for Chronic Musculoskeletal Pain Conditions: A Systematic Review and Meta-Analysis. Arthritis \& Rheumatism, 6, 717-724.

Hall, C.D.; Miszko, T., \& Wolf, S. L. (2009b). Effects of Tai Chi Intervention on Dual-Task Ability in Older Adults: A Pilot Study. Arch Phsy Med Rehabil, 90, 525-529.

Hartman, C.A.; Manos, T.M.; Winter, C.; Hartman, D.M.; Li, B., \& Smith, J.C. (2000). Effects of Tai Chi training on function and quality of life indicators in older adults with osteoarthritis. JAGS, 48, 1553-59.

Hernández-Reif, M.; Field, T.M., \& Thimas, E. (2001). Attention Deficit Hyperactivity Disorder: benefits from Tai Chi. J Bodyw Mov Ther, 5(2), 120-123.

Hoffmann-Smith, K.A.; Ma, A.; Yeh, C.T.; Deguire, N.L., \& Smith, J.P. (2009). The Effect of $\mathrm{Tai} \mathrm{Chi}$ in Reducing Anxiety in an Ambulatory Population. Journal of Complementary and Integrative Medicine, 6(1), 1-12.

Hong, Y., \& Li, J. (2007). Biomechanics of Tai Chi: A review. Sport Biomechanics, 6(3), 453-464. 
Jiménez-Martín, P.J.; Meléndez-Ortega, A.; Albers, U.; López-Díaz, A. (2013). Beneficios del Tai Chi Chuan en la osteoartritis, el equilibrio y la calidad de vida. RICYDE. Rev. int. cienc. deporte. 32(9), 181-199. http://dx.doi.org/10.5232/ricyde2013.03206

Irwin, M.; Olmstead, R., \& Motivala, S. (2008). Improving Sleep Quality in Older Adults wiith Moderate Sleep Complaints: A Randomized Controlled Trial of Tai Chi Chih. SLEEP, 31(7), 1001-1008.

Jackson, B.D.; Wluka, A.E.; Teichtahl, A.J.; Morris, M.E., \& Cicuttini, F.M. (2004). Reviewing knee osteoarthritis -a biomechanical perspective. J Sci Med Sport, 7, 34757.

Jin, P. (1989). Changes in Heart Rate, Noradrenaline, Cortisol and Mood during Tai Chi. J Psychosom Res, 33(2), 197-206.

Jin, P. (1992). Efficacy of Tai Chi, Brisk Walking, Meditation, and Reading in Reducing Mental and Emotional Stress. J Psychosom Res, 36(4), 361-370.

Kang, JW., Lee, MS, Posadzki, P., \& Ernst, E. (2011). T'ai chi for the treatment of osteoarthritis: a sistematic review and meta-analysis, BMJ Open, 2: 1-8,

Lelard, T.; Doutrellot, P.L.; David, P., \& Ahmaidi, S. (2010). Effects of a 12-Week Tai Chi Chuan Program Versus a Balance Training Program on Postural Control and Walking Ability in Older People. Arch Phsy Med Rehabil, 91, 9-14.

Lee, H.J.; Park, H.J.; Chae, Y.; Kim, S.Y.; Kim, S.N.; Kim, S.T.; Kim, J.H.; Yin, C.S., \& Lee, H. (2009). Tai Chi Qigong for the quality of life of patients with knee osteoarthritis: a pilot, randomized, waiting list controlled trial. Clin Rehab, 23, 504511.

Lee, M.S.; Lam, P., \& Ernst, E. (2008a). Effectiveness of tai chi for Parkinson's disease a critical review. Parkinsonism and Related Disorders, 14, 589-594.

Lee, M.S.; Pittler, M.H., \& Ernst, E. (2008b). Tai Chi for osteoarthritis: a systematic review. Clin Rheumatol, 27, 211-218.

Li, F.; McAuley, E.; Harmer, P.; Duncan, T.E., \& Cahumeton, N.R. (2001). Tai Chi Enhances Self-Efficacy and Exercise Behavoir in Older Adults. JAPA, 9(2), 161-171.

Li, F.; Fisher, K.J.; Harmer, P.; Irbe, D.; Tearse, R.G., \& Weimer, C. (2004). Tai Chi and Self-Rated Quality of Sleep and Daytime Sleepiness in Older Adults: A Randomized Controlled Trial. J. American Geriatrics Society, 52 (6), 892-900.

Li, F.; Harmer, P.; Fitzgerald, K.; Eckstrom, E.; Sotck, R.; Galver, J.; Maddalozzo, G., \& Batya, S. (2012). Tai Chi and Postural Stability in Patients with Parkinson's Disease, The New England J ournal of Medicine, 366(6), 511-519.

Li, J.X.; Xu, D.Q., \& Hong, Y. (2009a). Changes in muscle strength, endurance, and reaction of the lower extremities with Tai Chi intervention. Journal of Biomechanics, 42, 967-971.

Li, J.Y.; Zhang, Y.F.; Smith, G.S.; Xue, C.J.; Luo, Y.N.; Chen, W.H.; Skinner, C.J., \& Finkelstein, J. (2009b). Quality of Reporting of Randomized Clinical Trials in Tai Chi Interventions -A Systematic Review. Ecam Advance Access published, 7, 1-10.

Liu, X.; Miller, D.; Burton, N. W., \& Brown, W.J. (2010). A preliminary study of the effects of Tai Chi and Qigong medical exercise on indicators of metabolic syndrome, glycaemic control, health-related quality of life, and psychological health in adults with elevated blood glucose. Br J Sports Med, 44(10), 704-709.

Logghe, I.H.; Verhagen, A.P.; Rademaker, A.C.; Bierma-Zeinstra, S.M.; van Rossum, E.; Faber, M.J., \& Koes, B. W. (2010). The effects of Tai Chi on fall prevention, fear of falling \& balance in older people: A meta-analysis. Preventive Medicine, 51, 222227.

Low, S.; Ang, L.; Goh, K., \& Chew, S. (2009). A systematic review of the effectiveness of Tai Chi on fall reduction among the elderly. Archives of Gerontology and Geriatrics, $48,325-331$. 
Jiménez-Martín, P.J.; Meléndez-Ortega, A.; Albers, U.; López-Díaz, A. (2013). Beneficios del Tai Chi Chuan en la osteoartritis, el equilibrio y la calidad de vida. RICYDE. Rev. int. cienc. deporte. 32(9), 181-199. http://dx.doi.org/10.5232/ricyde2013.03206

Mark, M., \& Ling, P. (2003). Mediolateral Sway in Single-Leg Stance Is the Best Discriminator of Balance Performance for Tai-Chi Practitioners. Arch Phys Med Rehabil, 84, 683-686.

McGibbon, C.A.; Krebs, D.A.; Parker, S.W.; Scarborough, D.M.; Wayne, P.M., \& Wolf, S.L. (2005). Tai Chi and vestibular rehabilitation improve vestibulopathic gait via different neuromuscular mechanisms: Preliminary report. BMC Neurology, 5(3), 1-12.

Martínez, L.F.; Santos, M.L., y Casimiro, A.J. (2009). Condición física y Salud: un modelo didáctico de sesión para personas mayores. Revista Internacional de Medicina y Ciencias de la Actividad Física y el Deporte, 9(34), 140-157.

Ni, G.X.; Song, L.; Yu, B.; Huang, C.H., \& Lin J.H. (2010). Tai Chi Improves Physical Function in Older Chinese Women with Knee Osteoarthritis. Journal of Clinical Rheumatology, 16(2), 64-67

Qin, L.; Choy, W.; Leung, K.; Leung, P.C.; Au, S.; Hung, W.; Dambacher, M., \& Chan, K. (2005). Beneficial effects of regular Tai Chi exercise on musculoskeletal system. J Bone Miner Metab, 23(2), 186-190.

Ramachandran, A.K.; Rosengren, K.S.; Yang, Y., \& Hsiao-Wecksler, E.T. (2007). Effect of Tai Chi on gait and obstacle crossing behaviours in middle-aged adults. Gait \& Posture, 26, 248-255.

Reid-Arndt, S.; Matsuda, S., \& Cox, C. (2001). Tai Chi effects on neuropsychological, motional, and physical functioning following cancer treatment: A pilot study. Complementary Therapies in Clinical Practice, doi: 10.1016/j.ctcp.2011.02.2005.

Robins, J.L.; McCain, N.L.; Gray, D.P.; Elswick, R.K.; Walter, J.M., \& McDade, E. (2006). Research on psychoneuroimmunology : Tai Chi as a Stress Management Approach for Individuals with HIV disease. Applied Nursing Research, 19(1), 2-9.

Rogers, C.; Larkey, L., \& Keller, C. (2009). A Review of Clinical Trials of Tai Chi and Qigong in Older Adults. West J Nurs Res, 31(2), 245-279.

Shen, C.L.; James, C.R.; Chyu, M.C.; Bixby, W.R.; Brismee, J.M.; Zumwalt, M.A., \& Poklikuha, G. (2008). Effects of Tai Chi on Gait Kinematics, Physical Function, and Pain in Elderly with Knee Osteoarthritis - A Pilot Study. The American Journal of Chinese Medicine, 36(2), 219-232.

Song, R.; Lee, E.O.; Lam, P., \& Bae, S.C. (2003). Effects of tai chi exercise on pain, balance, muscle strength, and perceived difficulties in physical functioning in older women with osteoarthritis: a randomized clinical trial. J Rehumatol, 30(9), 20392044.

Song, R.; Lee, E.O.; Lam, P., \& Bae, S.C. (2007). Effects of a Sun-style Tai Chi Exercise on Arthritic Symptoms, Motivation and the Performance of Health Behaviors in Women with Osteoarthritis. Journal of Korean Academy of Nursing, $37(2), 249-256$.

Soto, J.R.; Dopico, X.; Giraldez, M.A.; Iglesias, E., \& Tuimil, J.L. (2008). Three Months of Practice of Tai Chi Chuan Improve the Balance of People Older than 60 Years: Practical study. Fitness \& Performance, 7(5), 306-311.

Spirduso, W.; Francis, K., \& MacRae, P. (2005). Physical Dimensions of Aging. United States: Human Kinetics.

Taggart, H. (2002). Effects of Tai Chi Exercise on Balance, Functional Mobility, and Fear of Falling Among Older Women. Applied Nursing Research, 15(4), 235-242.

Taylor-Piliae, R.E., \& Coull, B.M. (2012). Community-Based Yang-Style Tai Chi is safe and feasible in chronic stroke: a pilot study. Clinical Rehabilitation, 28(2), 121-131. 
Jiménez-Martín, P.J.; Meléndez-Ortega, A.; Albers, U.; López-Díaz, A. (2013). Beneficios del Tai Chi Chuan en la osteoartritis, el equilibrio y la calidad de vida. RICYDE. Rev. int. cienc. deporte. 32(9), 181-199. http://dx.doi.org/10.5232/ricyde2013.03206

Taylor-Piliae, R.E., \& Froelicher, E. (2004). Measurement properties of Tai Chi exercise self-efficacy among ethnic Chinese with coronary heart disease risk factors: a pilot study. European J ournal of Cardiovascular Nursing, 3, 287-294.

Taylor-Piliae, R.E.; Haskell, W.L.; Waters, C., \& Froelicher, E.S. (2006). Change in perceived psychosocial status following a 12-week Tai Chi exercise programme. Journal of Advanced Nursing, 54(3), 313-329.

Taylor-Piliae, R.E.; Newell, K.A.; Cherin, R.; Lee, M.J.; King, A.C., \& Haskell, W.L. (2010). Effects of Tai Chi and Western Exercise on Physical and Cognitive Functioning in Healthy Community-Dwelling Older Adults. JAPA, 18(3), 261-279.

Thorton, E.; Sykes, K., \& Tang, W. (2004). Health benefits of Tai Chi exercise: improved balance and blood pressure in middle-aged women. Health Promotions International, 19 (1), 33-38.

Toda, M.; Den, R.; Hasegawa-Ohira, M., \& Morimoto, K. (2011). Influence of personal patterns of behavoir on the effects of Tai Chi: a pilot study. Environ Health Prev Med, 16, 61-63.

Tsai, P.F.; Beck, C.; Chang, J.Y.; Hagen, J.; Kuo, Y.F.; Roberson, P.K.; Rosengren, K.; Beuscher, L.; Doan, C.L., \& Anand, K.J. (2009). The Effect of Tai Chi on Knee Osteoarthritis Pain in Cognitively Impaired Elders: Pilot Study. Geriatric Nursing, 30 (2), 132-139.

Tsang, W.W.; Wong, V.S.; Fu, S.N., \& Hui-Chan, C.W. (2004). Tai Chi Improves Standing Balance Control Under Reduced or Conflicting Sensory Conditions. Arch Phys Med Rehabil, 85, 129-137.

Tsang, W., \& Hui-chan, C.W. (2006). Standing Balance After Vestibular Stimulation in Tai Chi-Practicing and Nonpracticing Healthy Older Adults. Arch Phys Med Reahil, 87, 546-553.

Venglar, M. (2005). Case Report: Tai Chi and Parkinsonism. Physiotherapy Research International, 10(2), 116-121.

Verhagen, A.; Immink, M.; Meulen A., \& Bierma-Zeinstra, S. (2004). The efficacy of Tai Chi Chuan in older adults: a systematic review. Family Practice, 21(1), 107-113.

Vignon, E.; Valat, J.P. ; Rossignol, M. ; Avouac, B. ; Rozenberg, S. ; Thoumie, P. ; Avouac, J. ; Nordin, M., \& Hilliquin, P. (2006). Osteoarthritis of the knee and hip and activity: a systematic international review and synthesis (OASIS). Joint Bone Spine, 73(4), 442-455.

Wang, C.; Collet, J.P., \& Lau, J. (2004). The Effect of Tai Chi on Health Outcomes in Patients With Chronic Conditions. Arch Inter Med, 164, 493-501.

Wang, C.; Schmid, C.H.; Hibberd, P.L.; Kalish, R.; Roubenoff, R.; Rones, R., \& McAlindon, T. (2009). Tai Chi is effective in treating knee osteoarthritis: a randomized controlled trial. Arthritis Care \& Research, 61(11), 1545-1553.

Wang, C.; Raveendhara, B.; Ramel, J.; Kupelnick, B.; Scott, T., \& Schmid, C.H. (2010). Tai Chi on psychological well-being: systematic review and meta-analysis. BMC: Complementary \& Alternative Medicine, 10, 1-16.

Wayne, P.M.; Kiel, D.P.; Krebs, D.E.; Davis, R.B.; Savetsky-German, J.; Connelly, M., \& Buring, J.E. (2007). The effects of Tai Chi on bone mineral density in postmenopausal women: a systematic review. Arch Phys Med Rehabil, 88, 673-80.

Wayne, P.M.; Krebs, D.E.; Wolf, S.L.; Gill-Body, K.M.; Scarborough, D.M.; McGibbon, C.A.; Kaptchuk, T.J., \& Parker, S.W. (2004). Can Tai Chi Improve Vestibulopathic Postural Control? Arch Phys Med Rehabil, 85(1), 142-152. 
Jiménez-Martín, P.J.; Meléndez-Ortega, A.; Albers, U.; López-Díaz, A. (2013). Beneficios del Tai Chi Chuan en la osteoartritis, el equilibrio y la calidad de vida. RICYDE. Rev. int. cienc. deporte. 32(9), 181-199. http://dx.doi.org/10.5232/ricyde2013.03206

Wolf, S.L.; Barnhart, H.X.; Kutner, N.G.; McNeely, E.; Coogler, C. \& Xu, T. (1996). Reducing frailty and falls in older persons: an investigation of Tai Chi and computerized balance training. J AGS, 44(5), 489-497.

Wong, A.M.; Lin, Y.C.; Chou, S.W.; Tang, F.T., \& Wong, P.I. (2001). Coordination Exercise and Postural Stability in Elderly People: Effect of Tai Chi Chuan. Arch Phys Med Rehabil, 82, 608-612.

Woo, J.; Hong, A.; Lau, E., \& Lynn, H. (2007). A randomised controlled trial of Tai Chi and resistance exercise on bone health, muscle strength and balance in community-living elderly people. Age Ageing, 36, 262-268.

Wu, G. (2008). Age-Related Differences in Tai Chi Gait Kinematics and Leg Muscle Electromyography: A Pilot Study. Arch Phys Med Rehabil, 89, 351-356.

Wu, G.; Keyes, L.; Callas, P.; Ren, X., \& Bookchin, B. (2010). Comparison of Telecommunication, Community, and Home-Based Tai Chi Exercise Programs on Compliance and Effectiveness in Elders at Risk of Falls. Arch of Physical Medicine and Rehabilitation, 91(6), 849-856.

Xu, D.; Hong, Y., \& Chan, K. (2004). Effect of tai chi exercise on propioception of ankle and knee joints in old people. Br J Sports Med, 38, 50-54.

Yan, J.H. (1998). Tai Chi Improves Senior Citizen's Balance and Arm Movement Control. JAPA, 6, 271-284.

Yeh, G.Y.; Mietus, J.E.; Peng, C.K.; Phillips, R.S.; Davis, R.B.; Wayne, P.M.; Goldberger, A.L., \& Thomas, R. J. (2008). Enhancement of sleep stability with Tai Chi exercise in chronic heart failure: Preliminary findings using an ECG-based spectrogram method. Sleep Medicine, 9(5), 527-536.

Yeh, G.; Wang, C.; Wayne, P., \& Phillipis, R. (2009). Tai Chi Exercise for Patients with Cardiovascular Condictions and Risk Factors: A Systematic Review. Journal Cardiopul Rehabil Prev, 29(3), 152-160.

Zhang, J.G.; Takata, K.I.; Yamazaki, H.; Morita, T., \& Ohta, T. (2006). The effects of Tai Chi Chuan on physiological function and fear of falling in the less robust elderly: An intervention study for preventing falls. Archives of Gerontology and Geriatrics, 42(2), 107-116. 